\title{
Nonlinear Dynamics in a Chemical Reaction under an Amplitude-Modulated Excitation: Hysteresis, Vibrational Resonance, Multistability, and Chaos
}

\author{
A. V. Monwanou, ${ }^{1}$ A. A. Koukpémèdji, ${ }^{1,2}$ C. Ainamon, ${ }^{1}$ P. R. Nwagoum Tuwa, ${ }^{1,3,4}$ \\ C. H. Miwadinou $\mathbb{D}^{1,5}$ and J. B. Chabi Orou ${ }^{1}$ \\ ${ }^{1}$ Laboratoire de Mécanique des Fluides, de la Dynamique Nonlinéaire et de la Modélisation des Systèmes \\ Biologiques (LMFDNMSB), Institut de Mathématiques et de Sciences Physiques, Porto-Novo, Benin \\ ${ }^{2}$ Département de Physique, FAST-Natitingou, Université Nationale des Sciences, Technologies, Ingénierie et Mathématiques, \\ Abomey, Benin \\ ${ }^{3}$ Laboratory of Modelling and Simulation in Engineering, Biomimetics and Prototypes and TWAS Research Unit, \\ Faculty of Science, University of Yaoundé I, P. O. 812, Yaoundé, Cameroon \\ ${ }^{4}$ Research Unit of Industrial Systems Engineering and Environment (RU-ISEE), Fosto-Victor University Institute of Technology, \\ University of Dschang, P. O. Box 134, Bandjoun, Cameroon \\ ${ }^{5}$ Département de Physique, ENS-Natitingou, Université Nationale des Sciences, Technologies, Ingénierie et Mathématiques, \\ Abomey, Benin
}

Correspondence should be addressed to C. H. Miwadinou; clement.miwadinou@imsp-uac.org

Received 9 August 2020; Revised 29 August 2020; Accepted 17 September 2020; Published 5 October 2020

Academic Editor: Alina Gavrilu

Copyright (c) 2020 A. V. Monwanou et al. This is an open access article distributed under the Creative Commons Attribution License, which permits unrestricted use, distribution, and reproduction in any medium, provided the original work is properly cited.

\begin{abstract}
This paper deals with the effects of an amplitude-modulated (AM) excitation on the nonlinear dynamics of reactions between four molecules. The computation of the fixed points of the autonomous nonlinear chemical system has been made in detail using the Cardan's method. Hopf bifurcation has been also successfully checked. Routes to chaos have been investigated through bifurcations structures, Lyapunov exponent, phase portraits, and Poincaré section. The effects of the control force on chaotic motions have been strongly analyzed, and the control efficiency is found in the cases $g=0$ (unmodulated case) and $g \neq 0$ with $\Omega=\omega$ and $\Omega / w \neq p / q ; p$ and $q$ are simple positive integers. Vibrational resonance (VR), hysteresis, and coexistence of several attractors have been studied in detail based on the relationship between the frequencies of the AM force. Results of analytical investigations are validated and complemented by numerical simulations.
\end{abstract}

\section{Introduction}

Nonlinear dynamics is a multidisciplinary field that covers not only mathematics but also engineering, physics, chemistry, and biology. A monograph, authored by Strogatz [1], would be the first place to start if one is interested in learning about this topic. In the past decade, the study of complex dynamics and chemistry of oscillating reaction under the influence of external perturbation received much attention and various results such as period-doubling bifurcation leading to chaotic motion, quasiperiodic route to chaos, coexistence of multiple attractors, hysteresis, and vibrational resonance have been obtained [2-12]. The chemical oscillating reactions in a continuously stirred tank reactor (CSTR) is one of the first biochemical oscillations discovered. For considerable theoretical progress on the nature of chemical oscillation, the only known chemical oscillators were either biological in origin, like the glycolytic and oxidase-peroxidase systems; either discovered accidentally, like the Bray and BZ reactions; or variants of those reactions [2-12]. In these chemical oscillations, various dynamics behaviors are studied by many researchers. For 
instance, nonequilibrium phenomena such as oscillations, bistability, complex oscillations, and quasichaotic behavior of the reaction are revealed by these studies. One of the main challenges has been to predict and to control these phenomena in nonlinear chemical oscillations for potential applications (see [2-15]). The study of these oscillations has been made with a periodically external excitation or a parametric excitation $[11,12]$. Moreover, the study of these oscillations is more advantageous for systems with the AM (amplitude modulation) [14]. However, some new dynamical phenomena including controllable frequency can be also presented. Binous and Bellagi [2] present the solution of four problems drawn from the chemical and biochemical engineering field of study. These problems illustrate various important aspects of nonlinear dynamics such as limit cycles, quasiperiodic and chaotic behaviors, time series and phase portraits, power spectra, the time-delay reconstruction diagrams, Hopf bifurcation, bifurcation diagrams, and steady state multiplicity. Among a large number of examples, they have selected the following case studies because it illustrates the basic nonlinear dynamics concepts: the glycolytic oscillator model first suggested by Selkov in 1968 in order to elucidate the mechanism that living cells use to obtain energy from sugar breakdown; the oregonator model derived by Field, Koros, and Noyes in the early 1970s, which elucidates the famous oscillatory behavior observed in the Belousov-Zhabotinsky (BZ) reaction; the steady state multiplicity in a biochemical reactor for both the Monod and substrate inhibition kinetics; and the three-variable autocatalator, first proposed by Peng, Scott, and Showalter in 1990. Guruparan et al. [3] considered Brusselator chemical system driven by an amplitude-modulated (AM) force and studied numerically the dynamics of Brusselator chemical system driven by an amplitude-modulated force with widely different frequencies. They showed the occurrence of hysteresis and vibrational resonance and the coexistence of several period-T orbits, bifurcations of them, routes to chaos, and quasiperiodic and chaotic orbits. They have characterized periodic orbits, quasiperiodic orbits, chaotic orbits, hysteresis, and vibrational resonance using bifurcation diagram, maximal Lyapunov exponent, phase portrait, Poincaré map, and resonance plots. Shabunin et al. [5] introduced the lattice limit cycle (LLC) model as a minimal mean-field scheme which can model reactive dynamics on lattices (low-dimensional supports) producing nonlinear limit cycle oscillations. They have found that, under the influence of an external periodic force, the dynamics of the LLC may be drastically modified. Synchronization phenomena, bifurcations, and transitions to chaos are also observed as a function of the excitation force. Taking advantage of the drastic change in the dynamics due to the periodic forcing, they found that it is possible to modify the output/product or the production rate of a chemical reaction at will, simply by applying a periodic force to it, without the need to change the support properties or the experimental conditions. Blekhman and Landa [6] considered resonances caused by a biharmonical external force with two different frequencies (the so-called vibrational resonances) using a bistable oscillator described by a Duffing equation as an example. It is shown that, in the case of a weakly damped oscillator, these resonances are conjugate; they occur as either the low and high frequency is varied. In addition, the resonances occur as the amplitude of the high-frequency excitation is varied. It is also shown that the high-frequency action induces the change in the number of stable steady states; these bifurcations are also conjugate and are the cause of the seeming resonance in an overdamped oscillator. RoyLayinde et al. [7] examined the phenomenon of vibrational resonance (VR) and analyzed in a biharmonically driven two-fluid plasma model with nonlinear dissipation. They derived analytically an equation for the slow oscillations of the system in themes of the parameters of the fast signal using the method of direct separation of motion. The presence of a high-frequency externally applied electric field is found to significantly modify the systems dynamics and, consequently, induce VR. They have identified the origin of the VR in the plasma model, not only from the effective plasma potential but also from the contributions of the effective nonlinear dissipation. Besides several dynamical changes, including multiple symmetry-breaking bifurcations, attractor escapes, and reversed period-doubling bifurcations, numerical simulations also revealed the occurrence of single and double resonances induced by symmetry-breaking bifurcations. Landa and McClintock [8] considered the effect of a high-frequency force on the response of a bistable system to a low-frequency signal for both the overdamped and weakly damped cases. They have shown that the response can be optimized by an appropriate choice of vibration amplitude. This vibrational resonance displays many analogies to the well-known phenomenon of stochastic resonance, but with the vibrational force filling the role usually played by noise. Jeevarathinam et al. [9] analyzed the vibrational resonance in the Duffing oscillator system in the presence of a gamma-distributed time-delayed feedback and an integrative time-delayed (uniformly distributed time delays over a finite interval) feedback. Particularly, applying a theoretical procedure, they obtain an expression for the response amplitude at the low frequency of the driving biharmonic force. For both double-well potential and single-well potential cases, they are able to identify the regions in parameter space where either two resonances, a single resonance, or no resonance occur. Theoretically predicted values of the response amplitude and the values of a control parameter at which resonance occurs are in good agreement with their numerical simulation. The analysis shows a strong influence of both types of timedelayed feedback on vibrational resonance. In the present paper, we seek hysteresis, vibrational resonance, and chaos in the system of reactions between four molecules when it is subjected to an external amplitude modulation excitation. More precisely, after an in-depth analysis of the fixed points and of the Hopf bifurcation for the autonomous system, the effects of the modulated amplitude force on the dynamics of the chemical reaction considered have been studied in detail and the efficiency of the control force was analyzed. The paper is structured as follows: Section 2 gives the mathematical modeling of chemical model, while Section 3 analyses the fixed points and their stability and the possibility to 
obtain the Hopf bifurcation. In Section 4, in-depth details of the bifurcation and the route to chaos when the system is under the periodically external excitation are presented. Section 5 deals with vibrational resonance, bifurcation, route to chaos, bistability, coexistence of attractors, and hysteresis for $\Omega \gg \omega$. Section 6 analyses the effect of AM excitation when $\Omega=\omega$ and $\Omega / w \neq p / q$, where $p$ and $q$ are simple positive integers. Finally, the conclusion of the research is given in Section 7.

\section{Chemical Model and Its Mathematical Equations}

In this work, we consider the autonomous system of reactions between four molecules as in $[5,16]$ :

$$
2 X+2 Y \stackrel{k_{1}}{\longrightarrow} 3 Y+S, X+S \stackrel{k_{2}}{\longrightarrow} 2 Y, Y+S \stackrel{k_{3}}{\longrightarrow} 2 S .
$$

$X, Y$, and $S$ are molecules of the types $X$ and $Y$ and empty lattice sites, respectively, while $k_{1}, k_{2}$, and $k_{3}$ are kinetic constants of the corresponding reactions. The complex oscillatory dynamics and the formation of spatial patterns observed experimentally have been predicted with great success by reactive multimolecular patterns $[5,12,16-21]$. Indeed, at the beginning of the 80 years, experimentally, "vacation models" were written by reactive quadrimolecular stages. In these models, increasing the number of vacant sites results in an increase in autocatalytic behavior, which results in oscillatory behavior. The reactions between nitrogen monoxide and carbon monoxide $(\mathrm{NO}+\mathrm{CO})$, nitrogen monoxide and ammonia $\left(\mathrm{NO}+\mathrm{NH}_{3}\right)$, and nitrogen monoxide and dihydrogen $\left(\mathrm{NO}+\mathrm{H}_{2}\right)$, which all use the platinum surface $\mathrm{Pt}$ as catalyst, are well-known models of vacancies [18]. In each of these reactions, an autocatalytic behavior linked to vacant sites is observed, and this explains, amongst other nonlinear phenomena, the "surface explosion" phenomena observed frequently in heterogeneous catalysis representing narrow peaks of the product concentrations at regular temporal intervals $[5,12,16-21]$.

In the mean-field approach, the kinetic equations for the evolution of the relative concentrations of the molecules $(x$, $y$ ) and of empty sites (S) can be written as follows $[5,16]$ :

$$
\begin{aligned}
& \frac{\mathrm{d} x}{\mathrm{~d} t}=-2 k_{1} x^{2} y^{2}+k_{2} x s, \\
& \frac{\mathrm{d} y}{\mathrm{~d} t}=k_{1} x^{2} y^{2}-k_{3} y s, \\
& \frac{\mathrm{d} s}{\mathrm{~d} t}=2 k_{1} x^{2} y^{2}-k_{2} x s+k_{3} y s .
\end{aligned}
$$

It is easily shown that a condition of conservation is fulfilled:

$$
\frac{\mathrm{d} x}{\mathrm{~d} t}+\frac{\mathrm{d} y}{\mathrm{~d} t}+\frac{\mathrm{d} s}{\mathrm{~d} t}=0
$$

and hence, $x+y+s=$ cste. By choosing this constant equal to 1 , we have $x+y+s=1$, and putting it in equation (3), the system is reduced to the following system:

$$
\begin{aligned}
& \frac{\mathrm{d} x}{\mathrm{~d} t}=-2 k_{1} x^{2} y^{2}+k_{2} x(1-x-y), \\
& \frac{\mathrm{d} y}{\mathrm{~d} t}=k_{1} x^{2} y^{2}-k_{3} y(1-x-y) .
\end{aligned}
$$

The complex dynamic behaviors of this system under the action of a sinusoidal force have been studied in-depth $[5,16]$. Recently, the importance of the amplitude modulated force has been proven for the control of complex dynamics of certain systems such as mechanical and electrical systems $[14,15]$ and biochemical and chemical systems $[3,11-13]$. In this work, we consider the dynamics of molecules in the chemical reaction under the external amplitude-modulated excitation, which can be described by a system of two differential equations of order 1 . So we modify the original model (7) of the LLC oscillator by adding an amplitude modulation force to the right themes of the first equation. So, we have

$$
\begin{aligned}
\frac{\mathrm{d} x}{\mathrm{~d} t}= & -2 k_{1} x^{2} y^{2}+k_{2} x(1-x-y) \\
& +\left(F+2 G \cos \Omega_{0} t\right) \sin w_{0} t, \\
\frac{\mathrm{d} y}{\mathrm{~d} t}= & k_{1} x^{2} y^{2}-k_{3} y(1-x-y),
\end{aligned}
$$

where $F$ is the amplitude of unmodulated force, $G$ is the degree of modulation, and $w_{0}$ and $\Omega_{0}$ are the frequencies of the AMF.

Using the time rescaling $\tau=k_{1} t$ and notation $\dot{x}=\mathrm{d} x / \mathrm{d} \tau$, we reduce the system (8) as follows:

$$
\begin{aligned}
\dot{x}= & -2 x^{2} y^{2}+\alpha x(1-x-y) \\
& +(f+2 g \cos \Omega \tau) \sin \omega \tau \\
\dot{y}= & x^{2} y^{2}-\beta y(1-x-y),
\end{aligned}
$$

with $\quad \alpha=k_{2} / k_{1}>0 ; \beta=k_{3} / k_{1}>0, f=F / k_{1}, g=G / k_{1}$, $\Omega=\Omega_{0} / k_{1}$, and $w=w_{0} / k_{1}$.

\section{Equilibrium Points and Its Stability Analysis}

We consider the system (10) in the absence of the excitation $\mathrm{AM}$ and we seek the fixed points. Indeed, we have

$$
\begin{aligned}
-2 x^{2} y^{2}+\alpha x(1-x-y) & =0, \\
x^{2} y^{2}-\beta y(1-x-y) & =0 .
\end{aligned}
$$

The resolution of the system formed by these two equations comes down to that of the following equation:

$$
\frac{4 \beta^{2}}{\alpha^{2}} y^{4}-\beta y\left(1-\frac{2 \beta}{\alpha} y-y\right)=0 \text {. }
$$

The fixed points of the autonomous system are $E_{0}(0,0)$ and $E_{*}\left(x^{*}, y^{*}\right)$ whose coordinates $\left(x^{*}, y^{*}\right)$ are obtained after solving the following equation: 


$$
\frac{4 \beta^{2}}{\alpha^{2}} y^{3}-\beta\left(1-\frac{2 \beta}{\alpha} y-y\right)=0 .
$$

To solve equation (15), we set $p=\left((\alpha / 2)+\left(\alpha^{2} / 4 \beta\right)\right)$ and $q=-\alpha^{2} / 4 \beta$.

So, we have

$$
y^{3}+p y+q=0
$$

By taking the Cardan technique [11], the resolution of equation (16) amounts to looking for the roots of a trinome. If $\Delta$ is the discriminant of this trinomial, we have

$$
D=27 \Delta=4 p^{3}+27 q^{2} \text {. }
$$
$D>0$.

Here, $\alpha>0$ and $\beta>0$ so $p^{3}>0$ and $q^{2}>0$ and then

Equation (16) has only one real solution, which is $T=((-q \pm \sqrt{\Delta}) / 2)$. So, we have $E_{3}=\left(x_{3}, y_{3}\right)$, where

$$
\begin{aligned}
& x_{3}=\sqrt[3]{\frac{\beta^{2}}{\alpha}(1-\delta)}+\sqrt[3]{\frac{\beta^{2}}{\alpha}(1+\delta),} \\
& y_{3}=\sqrt[3]{\frac{\alpha^{2}}{8 \beta}(1-\delta)}+\sqrt[3]{\frac{\alpha^{2}}{8 \beta}(1-\delta)},
\end{aligned}
$$

$$
J=\left(\begin{array}{c}
-4 x^{*} y^{* 2}+\alpha\left(1-x^{*}-y^{*}\right)-\alpha x^{*} \\
2 x^{*} y^{* 2}+\beta y^{*}
\end{array}\right.
$$

For $E_{0}(0,0)$, the eigenvalues of the Jacobian $J$ are $\lambda_{1}=\alpha$ and $\lambda_{2}=-\beta$, which are reals and have opposite signs because $\alpha>0$ and $\beta>0$. Hence, $E_{0}$ is a saddle point. Then, the two eigenvalues of the Jacobian $J$ associated with $E_{1}(0,1)$ are $\lambda_{1}=0$ and $\lambda_{2}=\beta>0$, and therefore, $E_{1}$ is the unrobust point. On the contrary, for $E_{2}(1,0)$, the eigenvalues are $\lambda_{1}=0$ and $\lambda_{2}=-\alpha<0$, and therefore, $E_{2}$ is a robust point. Finally, for $E_{3}\left(x_{3}, y_{3}\right)$, the characteristic equation giving the eigenvalues of $J$ is

$$
\lambda^{2}+\sigma_{1} \lambda+\sigma_{2}=0
$$

with $\sigma_{1}=-\left(a_{1}+a_{2}\right)$ and $\sigma_{2}=a_{1} a_{4}-a_{2} a_{3}$, where

$$
\begin{aligned}
& a_{1}=-4 x_{3} y_{3}^{2}+\alpha\left(1-2 x_{3}-y_{3}\right), \\
& a_{2}=-4 x_{3}^{2} y_{3}-\alpha x_{3}, \\
& a_{3}=2 x_{3} y_{3}^{2}+\beta y_{3}, \\
& a_{4}=2 x_{3}^{2} y_{3}-\beta\left(1-x_{3}-2 y_{3}\right) .
\end{aligned}
$$

If $\sigma_{1}^{2}=4 \sigma_{2}$, then equation (21) has only one solution, and the fixed point $E_{3}$ is a center. For $\sigma_{1}^{2}>4 \sigma_{2}$, the eigenvalues are with

$$
\delta=\sqrt{\frac{(\alpha+2 \beta)^{3}}{27 \alpha \beta}+1}
$$

On the contrary, by combining the equations of the system (12), we have $x+y=1$.

Thus, we have $E_{1}(0,1)$ and $E_{2}=(1,0)$.

In total, the autonomous system admits exactly four fixed points $E_{0}(0,0)$ (trivial), $E_{1}(0,1)$ (semitrivial), $E_{2}(1,0)$ (semitrivial), and $E_{3}\left(x_{3}, y_{3}\right)$ (nontrivial).

The study of the stability of each fixed point is made by searching the Jacobian of the autonomous system associated. The Jacobian of the autonomous system associated with each fixed point $E_{0}(0,0)$ or $E_{*}\left(x^{*}, y^{*}\right)$ is as follows:

$$
\begin{aligned}
& \left.\begin{array}{c}
-4 x^{* 2} y^{*}-\alpha x^{*} \\
2 x^{* 2} y^{*}-\beta\left(1-x^{*}-y^{*}\right)+\beta y^{*}
\end{array}\right) . \\
& \begin{array}{l}
\lambda_{1}=\frac{-\sigma_{1}-\sqrt{\sigma_{1}^{2}-4 \sigma_{2}}}{2}, \\
\lambda_{2}=\frac{-\sigma_{1}+\sqrt{\sigma_{1}^{2}-4 \sigma_{2}}}{2} .
\end{array}
\end{aligned}
$$

In this case, the equilibrium point $E_{3}$ is a node. If $\sigma_{1}^{2}<4 \sigma_{2}$, then

$$
\begin{aligned}
& \lambda_{1}=\frac{-\sigma_{1}-i \sqrt{\sigma_{1}^{2}+4 \sigma_{2}}}{2}, \\
& \lambda_{2}=\frac{-\sigma_{1}+i \sqrt{-\sigma_{1}^{2}+4 \sigma_{2}}}{2},
\end{aligned}
$$

and $E_{3}$ is either a focus or a center. By virtue of the Routh-Hurwitz criterion $[22,23]$, the fixed point $E_{3}$ is stable if and only if $\sigma_{1}>0$ and $\sigma_{2}>0$ if not the fixed point is unstable. We now look for the Hopf bifurcation assuming that $\lambda=i w(w>0)$. Let us insert $\lambda$ in equation (21), and we have

$$
-w^{2}+i \sigma_{1} w+\sigma_{2}=0
$$

with $w=\sqrt{\sigma_{2}}$ and $\sigma_{1}=a_{1}+a_{4}=0$. 
Assuming $\beta$ fixed and taking $\alpha$ as a bifurcation parameter, differentiating both sides of the characteristic equation (21) associated to $E_{3}$ with respect to $\alpha_{H}$, we obtain

$$
\begin{aligned}
\frac{\mathrm{d} \lambda}{\mathrm{d} \alpha} & =-\frac{1}{2} \frac{\mathrm{d} \sigma_{1}}{\mathrm{~d} \alpha}-\frac{1}{2 \lambda} \frac{\mathrm{d} \sigma_{2}}{\mathrm{~d} \alpha}, \\
R_{e}\left(\frac{\mathrm{d} \lambda}{\mathrm{d} \alpha}\right) & =-\frac{1}{2} \frac{\mathrm{d} \sigma_{1}}{\mathrm{~d} \alpha},
\end{aligned}
$$

where $\alpha=\alpha_{H}$ and $\lambda=i w$. Thus, if for $\alpha=\alpha_{H}, \sigma_{1}=0$, and $R_{e}(\mathrm{~d} \lambda / \mathrm{d} \alpha) \neq 0$, then $\alpha=\alpha_{H}$ is a Hopf bifurcation value for the equilibrium point system $E_{3}$. In short, we have the following Theorem 1.

Theorem 1. If $\beta$ is fixed, system (8) in absence of the $A M$ force has a Hopf bifurcation at equilibrium point $E_{3}\left(x_{3}, y_{3}\right)$ when $\alpha$ passes through the critical value $\alpha_{H}$, which verifies simultaneously the following relations (16 and 17):

$$
\begin{aligned}
& \alpha-\beta-\frac{1}{2} \alpha\left[\frac{\alpha^{2}(1-\delta)}{\beta}\right]^{1 / 3}+\beta\left[\frac{\alpha^{2}(1-\delta)}{\beta}\right]^{1 / 3}-2 \alpha\left[\frac{\beta^{2}(1-\delta)}{\alpha}\right]^{1 / 3}+\beta\left[\frac{\beta^{2}(1-\delta)}{\alpha}\right]^{1 / 3} \\
& -\left[\frac{\alpha^{2}(1-\delta)}{\beta}\right]^{2 / 3}\left[\frac{\beta^{2}(1-\delta)}{\alpha}\right]^{1 / 3}+\left[\frac{\alpha^{2}(1-\delta)}{\beta}\right]^{1 / 3}\left[\frac{\beta^{2}(1-\delta)}{\alpha}\right]^{2 / 3} \\
& -\frac{1}{2} \alpha\left[\frac{\alpha^{2}(1+\delta)}{\beta}\right]^{1 / 3}+\beta\left[\frac{\alpha^{2}(1+\delta)}{\beta}\right]^{1 / 3}-2\left[\frac{\alpha^{2}(1-\delta)}{\beta}\right]^{1 / 3}\left[\frac{\beta^{2}(1-\delta)}{\alpha}\right]^{1 / 3}\left[\frac{\alpha^{2}(1+\delta)}{\beta}\right]^{1 / 3} \\
& +\left[\frac{\beta^{2}(1-\delta)}{\alpha}\right]^{2 / 3}\left[\frac{\alpha^{2}(1+\delta)}{\beta}\right]^{1 / 3}-\left[\frac{\beta^{2}(1-\delta)}{\alpha}\right]^{1 / 3}\left[\frac{\alpha^{2}(1+\delta)}{\beta}\right]^{2 / 3}-2 \alpha\left[\frac{\beta^{2}(1+\delta)}{\alpha}\right]^{1 / 3} \\
& +\beta\left[\frac{\beta^{2}(1+\delta)}{\alpha}\right]^{1 / 3}-\left[\frac{\alpha^{2}(1-\delta)}{\beta}\right]^{2 / 3}\left[\frac{\beta^{2}(1+\delta)}{\alpha}\right]^{1 / 3}+2\left[\frac{\alpha^{2}(1-\delta)}{\beta}\right]^{1 / 3}\left[\frac{\beta^{2}(1-\delta)}{\alpha}\right]^{1 / 3}\left[\frac{\beta^{2}(1+\delta)}{\alpha}\right]^{1 / 3} \\
& -2\left[\frac{\alpha^{2}(1-\delta)}{\beta}\right]^{1 / 3}\left[\frac{\alpha^{2}(1-\delta)}{\beta}\right]^{1 / 3}\left[\frac{\beta^{2}(1+\delta)}{\alpha}\right]^{1 / 3}+2\left[\frac{\alpha^{2}(1+\delta)}{\beta}\right]^{1 / 3}\left[\frac{\beta^{2}(1-\delta)}{\alpha}\right]^{1 / 3}\left[\frac{\beta^{2}(1+\delta)}{\alpha}\right]^{1 / 3} \\
& -\left[\frac{\alpha^{2}(1+\delta)}{\beta}\right]^{2 / 3}\left[\frac{\beta^{2}(1+\delta)}{\alpha}\right]^{1 / 3}+\left[\frac{\beta^{2}(1+\delta)}{\alpha}\right]^{2 / 3}\left[\frac{\alpha^{2}(1-\delta)}{\beta}\right]^{1 / 3}+\left[\frac{\beta^{2}(1+\delta)}{\alpha}\right]^{2 / 3}\left[\frac{\alpha^{2}(1+\delta)}{\beta}\right]^{1 / 3}=0 \text {, } \\
& \left(-4 y_{3}^{2}+4 x_{3} y_{3}-2 \alpha+\beta\right) \frac{\mathrm{d} x_{3}}{\mathrm{~d} \alpha}+\left(2 x_{3}^{2}-8 x_{3} y_{3}-\alpha+2 \beta\right) \frac{\mathrm{d} y_{3}}{\mathrm{~d} \alpha}-2 x_{3}-y_{3}+1 \neq 0 .
\end{aligned}
$$

To support this theorem, we represent the real part of the eigenvalues associated with the equilibrium point $E_{3}\left(x_{3}, y_{3}\right)$ in the case where the eigenvalues are complex as a function of $\alpha$ for fixed values of $\beta$. Figure 1 shows the critical values $\alpha_{H} \approx 0.00845, \alpha_{H} \approx 0.0237, \alpha_{H} \approx 0.059, \alpha_{H} \approx 0.0875$ of $\alpha$ corresponding to the Hopf bifurcation points $H_{1}, H_{2}, H_{3}$ and $H_{4}$ respectively. By a simple calculation, we show that each of these values verifies simultaneously (28) and (29). It should also be noted that the critical value $\alpha H$ for the Hopf bifurcation increases with $\beta$. At the moment of its birth, through Hopf bifurcation of the point $E_{3}\left(x_{3}, y_{3}\right)$, the oscillations have infinitesimally small amplitude and nearharmonic shape (see Figure 2).

\section{Bifurcation and Route to Chaos for $g=0$}

Before studying the influence of the AM excitation on the dynamics of the system, let us analyze the effect of each parameter $\alpha, \beta$, and $f$ on the dynamics of the unmodulated system. Indeed, we represent the bifurcation diagram, the Lyapunov exponent, the phase space, and the Poincare section taking as control parameters $f$ and $\beta$. In the remains of this work, we take $\omega=0.0481, \alpha>0, O>0, x(0)=0.5$, and $y(0)=0.5$. From all results of the simulations carried out in the case of the unmodulated system $(g=0)$, we note that the chemical reaction studied can present a very rich dynamic such as periodic, multiperiodic, quasiperiodic, and chaotic oscillations. In addition, we observe phenomena such as hysteresis, multistability, coexistence of periodic, multiperiodic, quasiperiodic, and chaotic attractors (see Figures 3-6). All these phenomena prove that very complex dynamics can be observed in the nonlinear chemical reaction studied. Thus, Figure 3 represents the bifurcation diagram and corresponding Lyapunov exponents for the system when $f$ varies in the domain $0 \leq f \leq 0.003$, the other parameters being fixed. From this figure, it emerges that the 


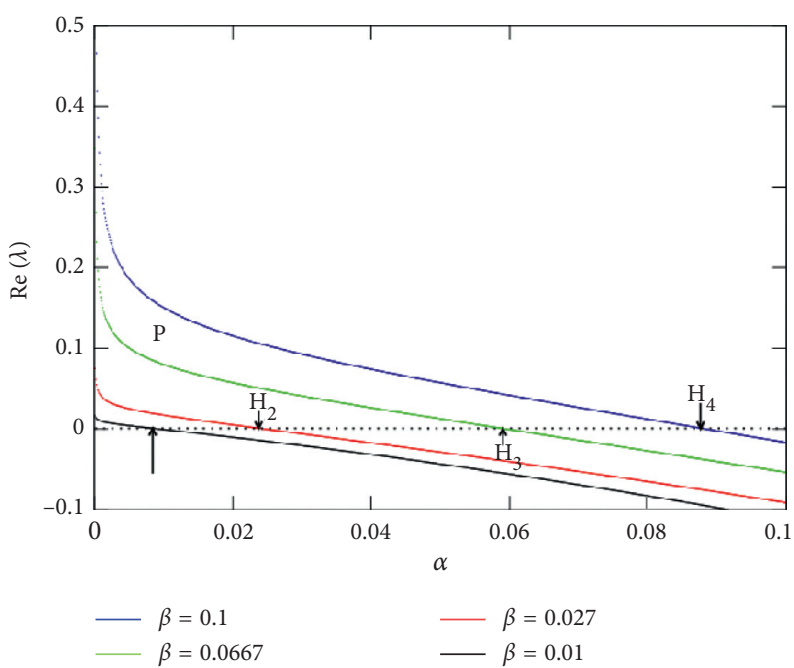

Figure 1: Real part of the eigenvalues versus $\alpha$ for different values of $\beta . H_{1}, H_{2}, H_{3}$ and $H_{4}$ are the points where Re $(\lambda)=0$.

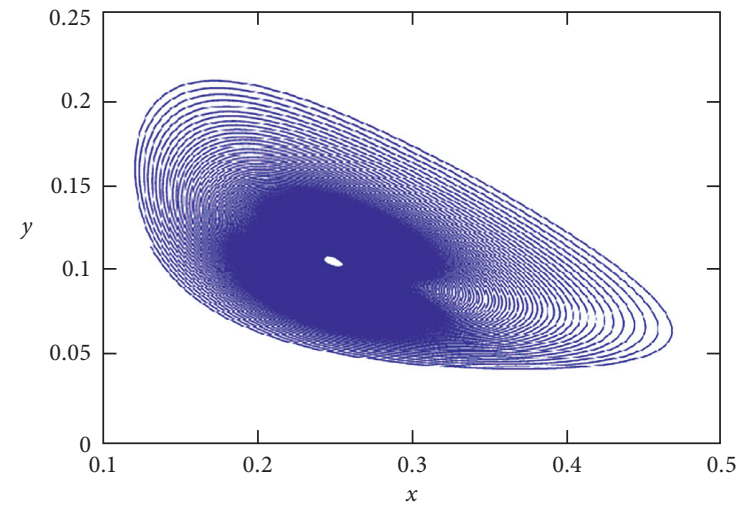

(a)

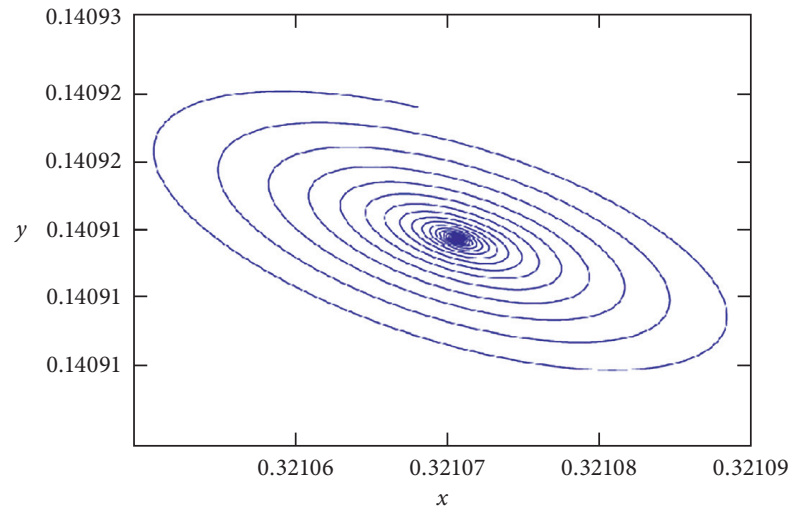

(b)

Figure 2: Phase portraits of the autonomous system for parameter values corresponding to Hopf bifurcation at fixed point $E_{3}=\left(x_{3}, y_{3}\right)(\mathrm{a})$ : $\alpha_{H}^{\prime}=0.00845 ; \beta=0.01$ and $(\mathrm{b}): \alpha_{H}^{\prime}=0.0237 ; \beta=0.027$.

chemical oscillations are quasiperiodic, multiperiodic, and chaotic. When we compare the dynamics of the system when $f$ increases from 0 to 0.003 (blue color) to that is obtained when $f$ decreases from 0.003 to 0 (red color), we note apart from chemical oscillations of the same nature which are observed on a large domain that the system presents the coexistence of the attractors of period $6 T$ with quasiperiodic attractors for $0.0011162<f<0.001131$, attractors of period $5 T$ with quasiperiodic attractors for $0.001521<$ $f<0.00156103$ and $0.001598 \leq f<0.0017485$, attractors of period $5 T$ with attractors of period $10 T$ for $0.00156103<$ $f<0.001598$, and attractors of period $5 T$ with chaotic attractors for $0.00298<f \leq 0.003$. The chaotic behavior of chemical oscillations predicted by the bifurcation diagram and its corresponding Lyapunov exponent (Figure 3) is confirmed by the phase space (Figure 4(a)) and the corresponding Poincaré section (Figure 4(b)) obtained for $f=0.0025$. For $f=0.00165$ and with two different initial conditions, we have plotted the phase space and the corresponding Poincaré section in Figure 5. We clearly note through this figure the coexistence of the quasiperiodic attractors (Figures 5(a) and 5(b)) with the attractors of period 5T (Figures 5(c) and 5(d)) thus justifying this phenomenon. To understand the influence of the nonlinear parameters $\alpha$ and $\beta$ on the nature of the oscillations of the chemical reaction considered, we consider now $\beta$ as the bifurcation parameter. Thus, Figure 6 shows the effect of the parameter $\alpha$ on the bifurcation diagram when $\beta$ varies between 0.03 and 0.1 . As it can be seen in this figure, the chaotic, periodic, multiperiodic, and quasiperiodic behaviors also depend on $\beta$. We take note that chaotic oscillations can only exist when $0.02 \leq \alpha<0.05$, and outside this domain, the chemical oscillations are periodic. It should be noted that the numerical simulations have shown that $\alpha$ and $\beta$ have almost the same effects on the chemical dynamics of the studied reaction. 


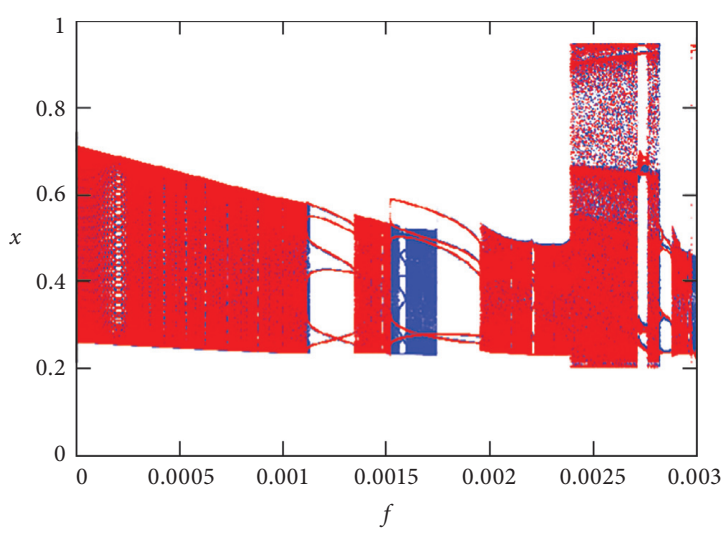

(a)

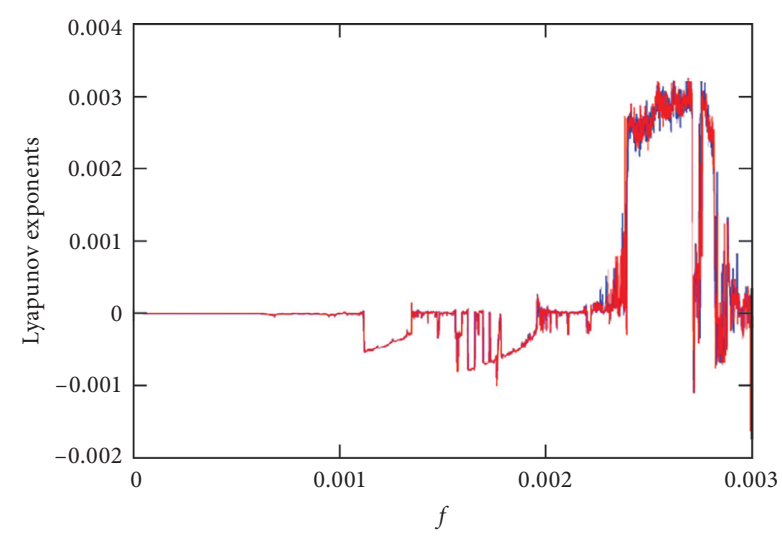

(b)

FIGURE 3: Bifurcation diagram and its corresponding Lyapunov exponent of nonlinear chemical reaction with $\omega=0.0481, \alpha=0.0413$, $\beta=0.0667$, and $g=0$. Bifurcation diagrams and its corresponding Lyapunov exponent are obtained by scanning the parameter $f$ upwards (blue) and downwards (red).

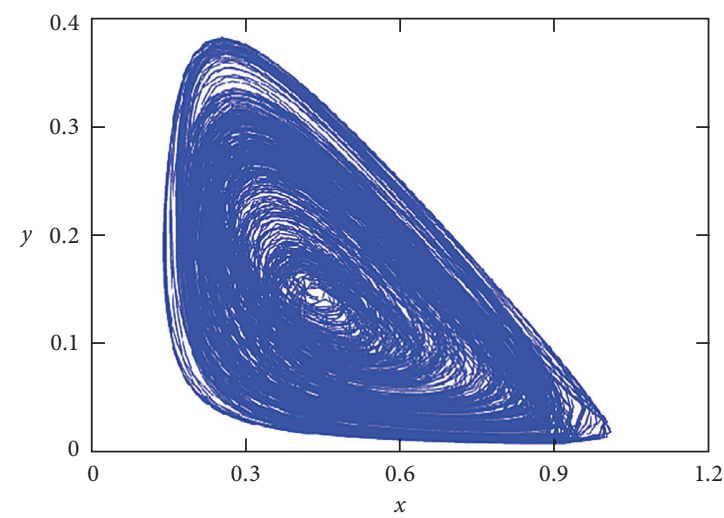

(a)

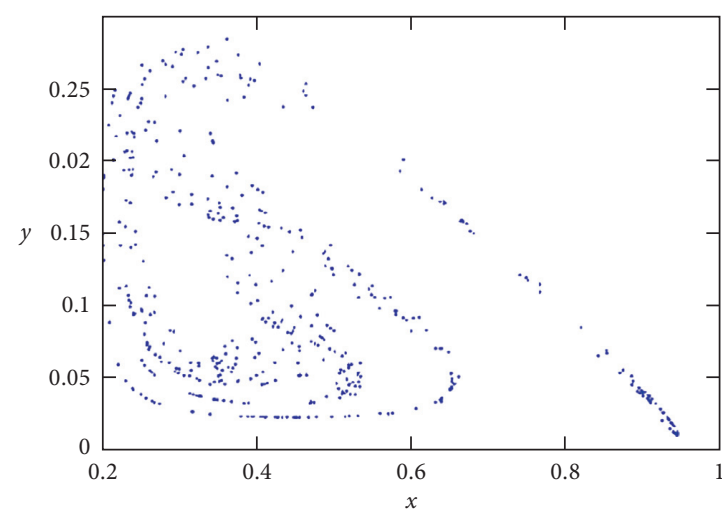

(b)

FIgURE 4: Chaotic phase portrait and its Poincaré section of nonlinear chemical reaction with parameters of Figure 3 and $f=0.0025$.

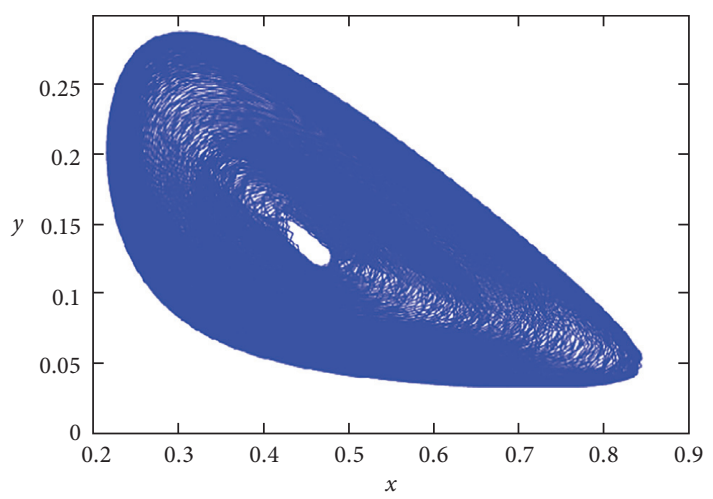

(a)

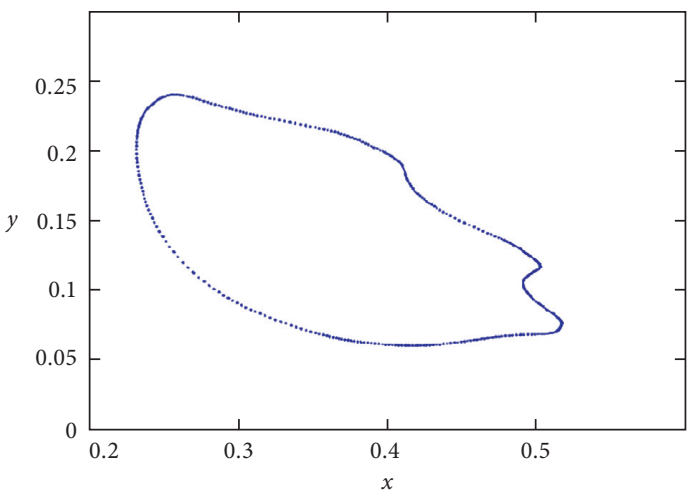

(b)

Figure 5: Continued. 


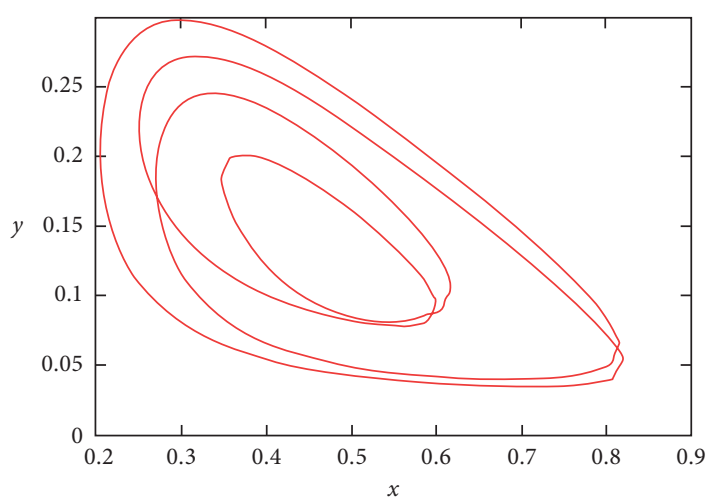

(c)

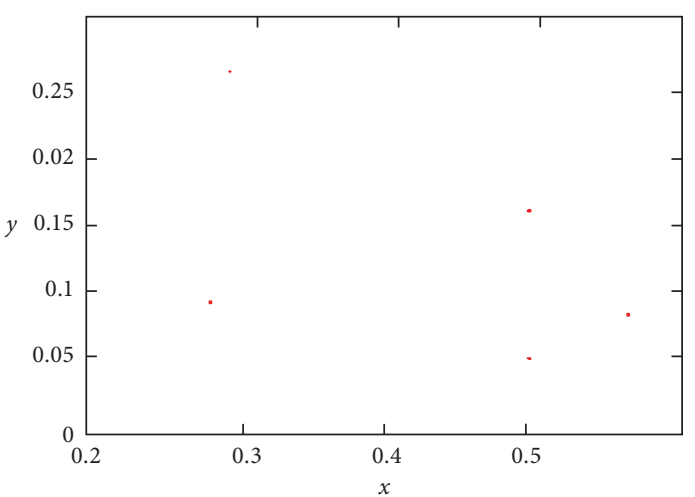

(d)

Figure 5: Phase space and its corresponding Poincaré section of nonlinear chemical reaction with $f=0.00165$ and other parameters of Figure 3. $(\mathrm{a}, \mathrm{b})$ Quasiperiodic attractor for initial conditions $(0.5,0.5)$ and $(\mathrm{c}, \mathrm{d})$ attractor of period $5 T$ for initial conditions $(0.1,0.1)$.

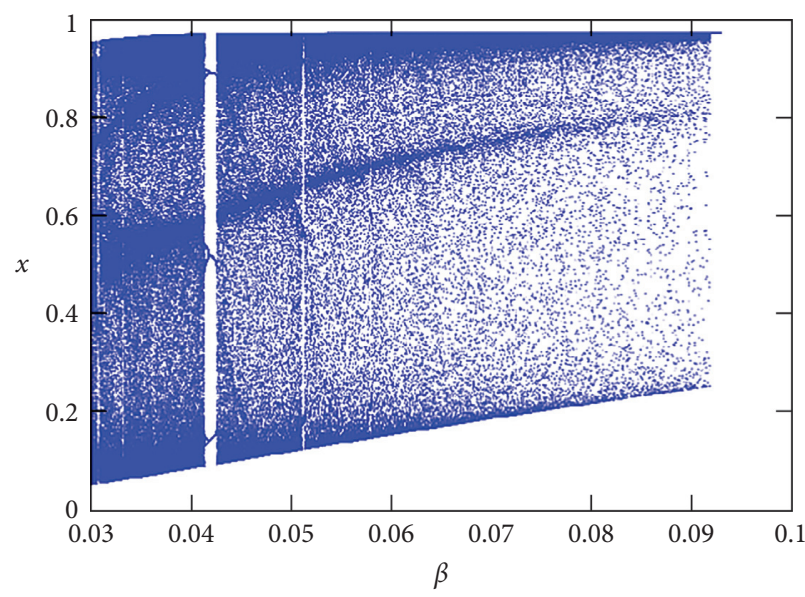

(a)

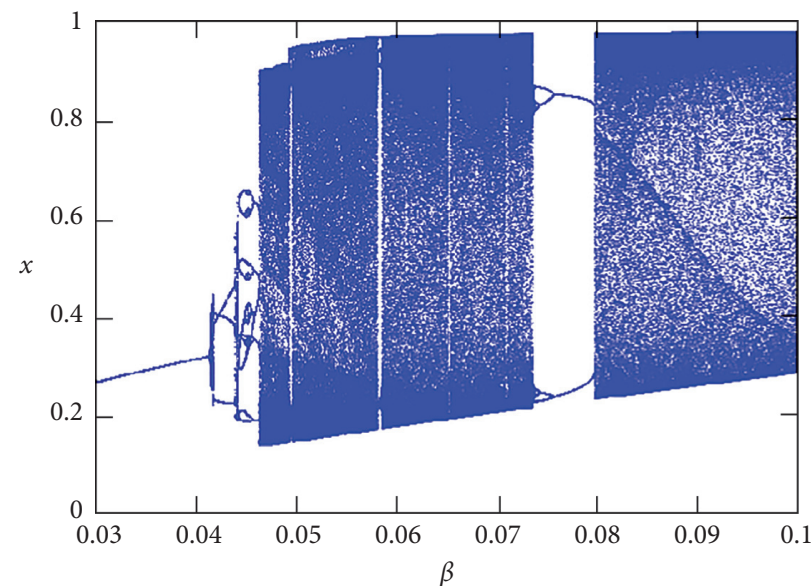

(b)

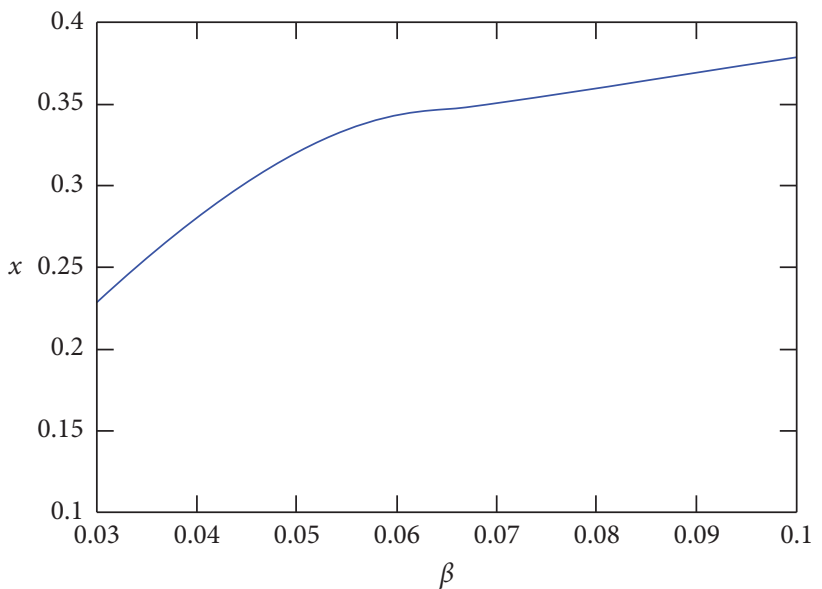

(c)

FiguRE 6: Effect of $\alpha$ on bifurcation diagram of nonlinear chemical reaction with $\omega=0.0481, f=0.00165$, and $g=0$ : (a) $\alpha=0.02$; (b) $\alpha=0.03$; (c) $\alpha=0.05$ 


\section{Effect of AM Force on the System with $\Omega \gg \omega$}

5.1. Vibrational Resonance and Amplitude Response. In a nonlinear dynamical system driven by a biharmonic force consisting of a low-frequency $\omega$ and a high-frequency $\Omega$ with $\Omega \omega$, when the amplitude $g$ of the high-frequency force is varied, the amplitude response at the low-frequency $\omega$ exhibits a resonance. This high-frequency force-induced resonance is called vibrational resonance [3, 6-9]. To determine the VR, we use the amplitude of the response at the frequency $\omega$ of the signal. Indeed, using the fourth-order Runge-Kutta algorithm, with time step size, we numerically integrate the system (10) of the chemical reaction under consideration. Thus, the numerical solution $x(\tau)$ allows to calculate the amplitude response $Q$ through the following formula:

$$
Q=\frac{\sqrt{Q_{s}^{2}+Q_{c}^{2}}}{f},
$$

where

$$
\begin{aligned}
& Q_{s}=\frac{2}{n \pi} \int_{0}^{n T} x(\tau) \sin w \tau \mathrm{d} \tau, \\
& Q_{c}=\frac{2}{n \pi} \int_{0}^{n T} x(\tau) \cos w \tau \mathrm{d} \tau,
\end{aligned}
$$

with $T=2 \pi / \omega$ the response period and $n=500$.

We compute $Q$ with a low-frequency force only, a highfrequency only, and with both forces.

For $f=0, Q$ is determined as $\sqrt{Q_{s}^{2}+Q_{c}^{2}}, Q_{s}$ and $Q_{c}$ and representing the Fourier coefficients of the output signal at the frequency $2 \pi T$ and $Q$ the amplitude of the response to this same frequency. Indeed, for $\Omega=80 \omega$ with $\omega$ being small, the different results are shown in Figures 7-9. Figures 7(a) and 7(b) represent the variation in $Q$ depending, respectively, on $f$ for $g=0$ and $g$ for $f=0$ when $\omega=0.0481$. From Figure 7(a), we note the presence of several peaks, and while $f=0$, we have a single maximum for which $g=0.003301$ and $Q=0.00092802$. When $g$ varies from 0 to 0.05 with $f 6=0$ and $\Omega=80 \omega$, the VR appears and we observe that its persistence, its form, its maximum amplitude, and the value of $g$ at the resonance depends not only on $f$ and $\omega$ but also on nonlinear parameters $\alpha$ and $\beta$ of the nonlinear chemical action considered. Indeed, a multiresonance appears for $\omega<0.06$ (see Figure 8(a)) and disappears when $0.06 \leq \omega<0.09$. For $0.09 \leq \omega \leq 0.1$, the VR becomes a double resonance with two different $\mathrm{Q}_{\max }$ (see Figure $8(\mathrm{~b})$ ) and disappears again when $0.1<\omega \leq 0.14$ and then reappears in the form of a monoresonance when $\omega>0.14$ (see Figure $8(\mathrm{c})$ ). Finally, we note that when $g$ varies from 0 to 0.05 , the maximum amplitude $\mathrm{Q}_{\max }$ decreases when the frequency $\omega$ increases.
Figure 9 shows when $g$ varies from 0 to $0.05, \Omega=80 \omega$ with $\omega=0.16$, and the influence of the parameters $f, \alpha$, are $\beta$ (Figures 9(a)-9(c), respectively) is shown on the VR. From Figure 9(a), we note that the maximum value of the amplitude of the response decreases when $f$ increases and we obtain a double resonance when $f=0.0011$. Figure 9(b) shows that the parameter $\alpha$ also influences the vibrational resonance. Finally, we notice through Figure 9(c) that VR exists when $0.06<\beta<0.08$ and that the parameter $\beta$ also has the same effects on VR as in the case of $\alpha$. In conclusion, the birth and the disappearance of the vibrational resonance in the chemical reaction studied can be strongly controlled not only by the AM force but also by the parameters $\alpha$ and $\beta$ of the chemical reaction.

5.2. Hysteresis, Coexistence of Attractors, and Multistability. In this section, we analyze the effect of $g$ on chemical dynamics when $\Omega \gg \omega$ by looking the hysteresis and coexistence of attractors phenomena. For this, we represent the bifurcation diagram by varying $g$ with $\Omega=80 \omega$ (Figure 10).

When we compare the dynamics of the system when $g$ increases from 0 to 0.1 (blue color, Figure 10(a)) to that obtained when $g$ decreases from 0.1 to 0 (red color, Figure 10(b)), we note that the chemical oscillations of the same kind are observed but with amplitudes that outside $\mathrm{x}$ different especially for multiperiodical oscillations. This difference in amplitude noted shows that the chemical oscillations do not follow the same path as going back and forth when we increase $g$ from 0 to 0.1 and when we decrease it from 0.1 to 0 : this phenomenon is called hysteresis. There also needs to be a domain where multiperiodic attractors coexist with attractors of period $2 \mathrm{~T}$ and attractors of period $4 T$ on the one hand and also the coexistence on the other hand of the chaotic attractors with the attractors of period $4 T$ and multiperiodic attractors. The phenomena of hysteresis and coexistence of attractors are very visible in Figure 10 (confirmed by Figure 11) and show multistable behaviors and hysteresis in the system. Thus, for $\Omega=80 \omega$, the chemical oscillations become more complex for the reaction considered. Figure 12 shows the effect of $\beta$ on the chemical oscillations and the two phenomena obtained. We deduce that when the parameter $\beta \in[0,0.06]$, the chemical oscillations are not chaotic and there is coexistence of periodic attractors and the presence of hysteresis.

The chemical oscillations become very varied, and we note periodic, multiperiodic, quasiperiodic, and chaotic behaviors with the presence and persistence of hysteresis and the coexistence of multiple attractors when $\beta \in[0.06,0.7]$. Finally, for $\beta>0.7$, we observe a total dominance of chaotic oscillations and the disappearance of the hysteresis phenomenon. The parameters $\alpha$ and $f$ have similar effects to that of $\beta$ reason for which we decided not to represent the figures 


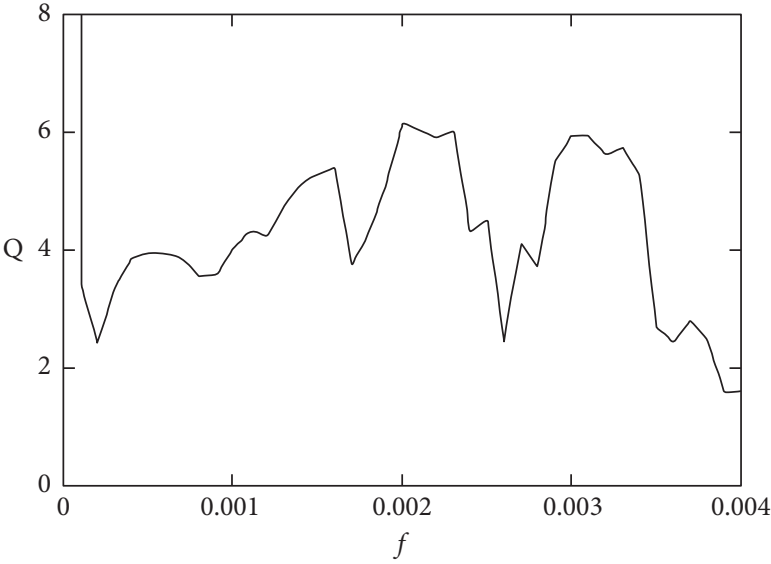

(a)

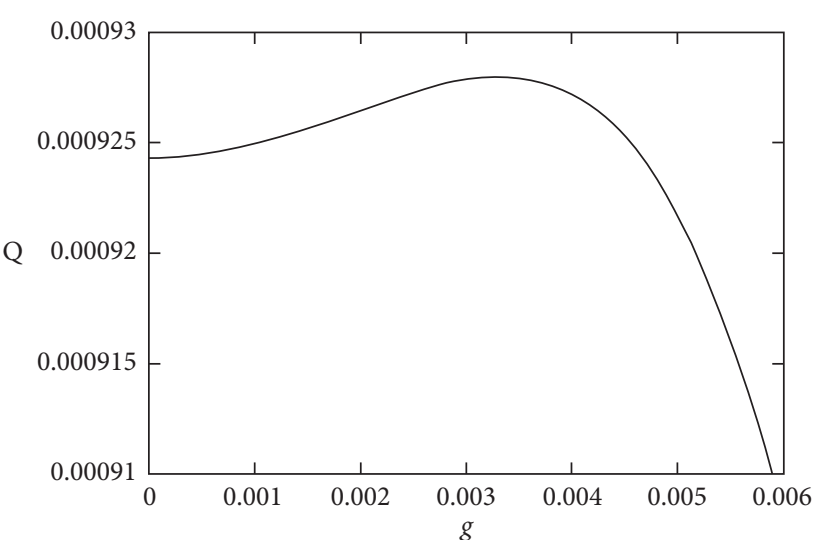

(b)

FIGURE 7: (a) Variation in numerically computed $Q$ against the control parameter $f$ with $\omega=0.0481, \alpha=0.0413, \beta=0.0667$, and $g=0$; (b) variation in numerically computed $Q$ against the control parameter $g$ with $\omega=0.0481, \Omega=80 \omega, \alpha=0.0413, \beta=0.0667$, and $f=0$.

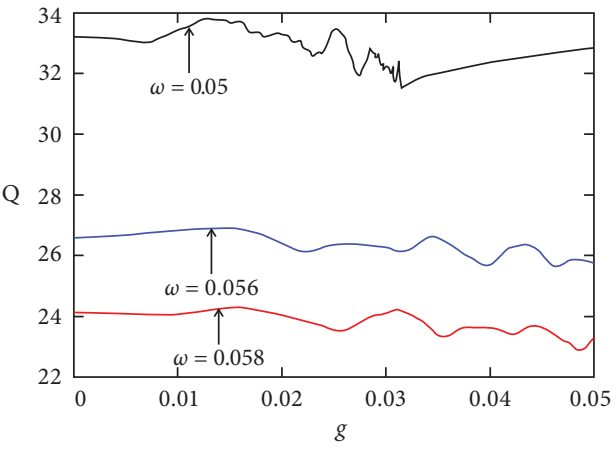

(a)

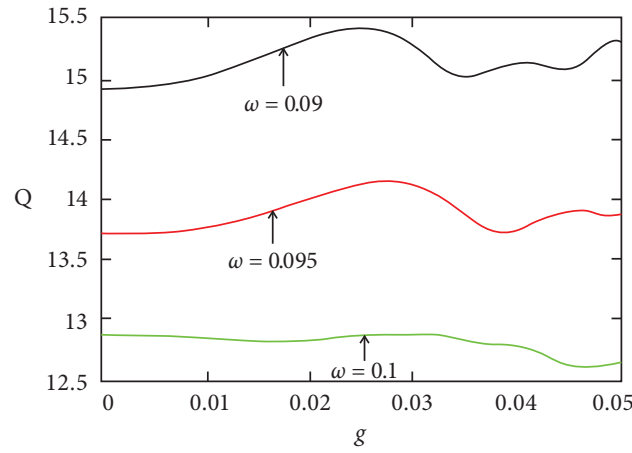

(b)

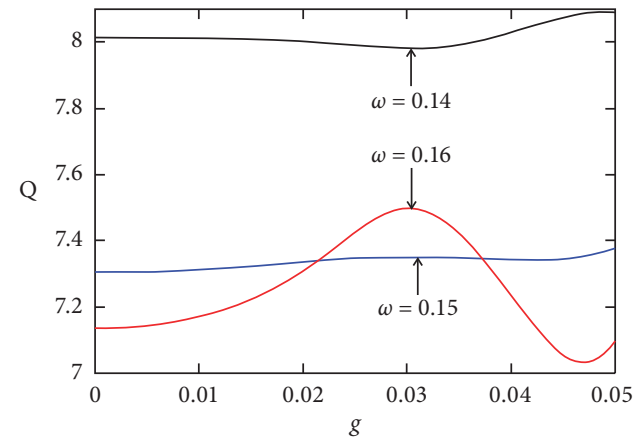

(c)

Figure 8: Effect of frequency $\omega$ on response amplitude Q versus g with $\omega=0.0481, \Omega=80 \omega, \alpha=0.0413, \beta=0.0667$, and $f=0.001$.

translating these effects. We notice that the parameter $g$ and the frequencies of the AM force have a very important effect on the dynamics of the system as well as on the phenomena of hysteresis and coexistence of attractors.

More precisely, $g$ can be used to reduce the domains where the oscillations are quasiperiodic, the domains of coexistence of attractors, and to make disappear completely hysteresis.

\section{Effect of AM Force in the System with $\omega=\Omega$}

Here, the effect of the AM force is analyzed in the case $\omega=\Omega$. For this reason, we chose $g$ as the bifurcation parameter, and the results obtained are shown in Figure 13. From the analysis of Figure 13, it appears that the variation in $\alpha$ can make disappear chaotic and quasiperiodic oscillations but preserves the phenomena of hysteresis and coexistence of 


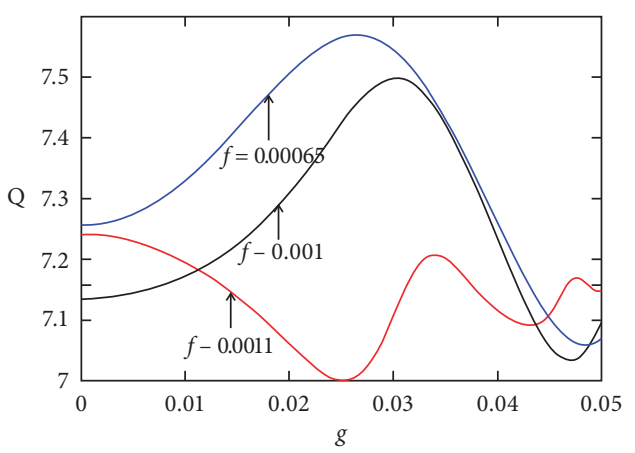

(a)

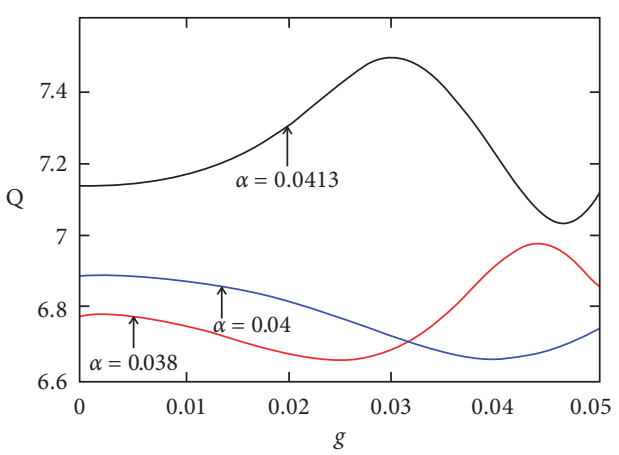

(b)

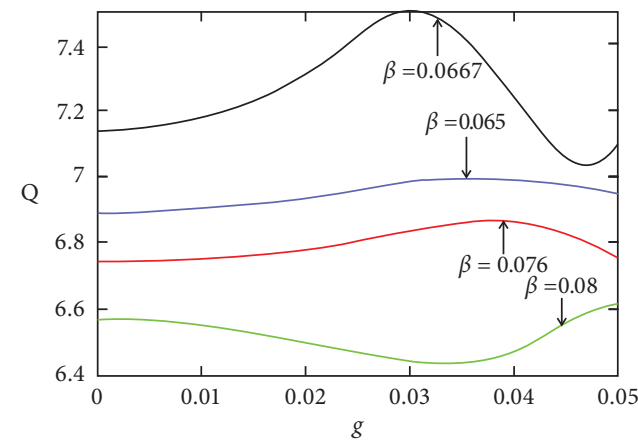

(c)

Figure 9: (a) Effect of amplitude $f$ on response amplitude $Q$ versus g with $\omega=0.016, \Omega=80 \omega, \alpha=0.0413$, and $\beta=0.0667$; (b) effect of nonlinear parameter $\alpha$ on response amplitude $Q$ versus $g$ with $\omega=0.016, \Omega=80 \omega, \beta=0.0667$, and $f=0.001$; (c) effect of nonlinear parameter $\beta$ on response amplitude $Q$ versus $g$ with $\omega=0.016, \Omega=80 \omega, \alpha=0.0413$, and $f=0.001$.

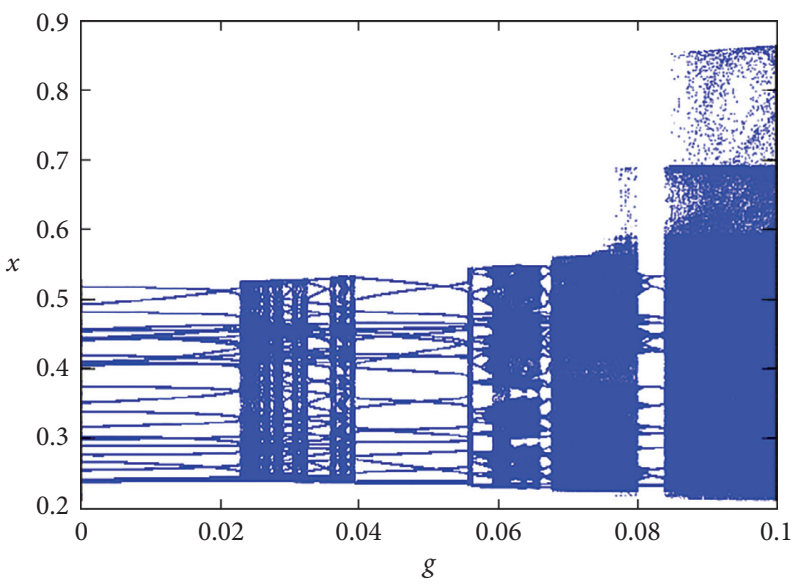

(a)

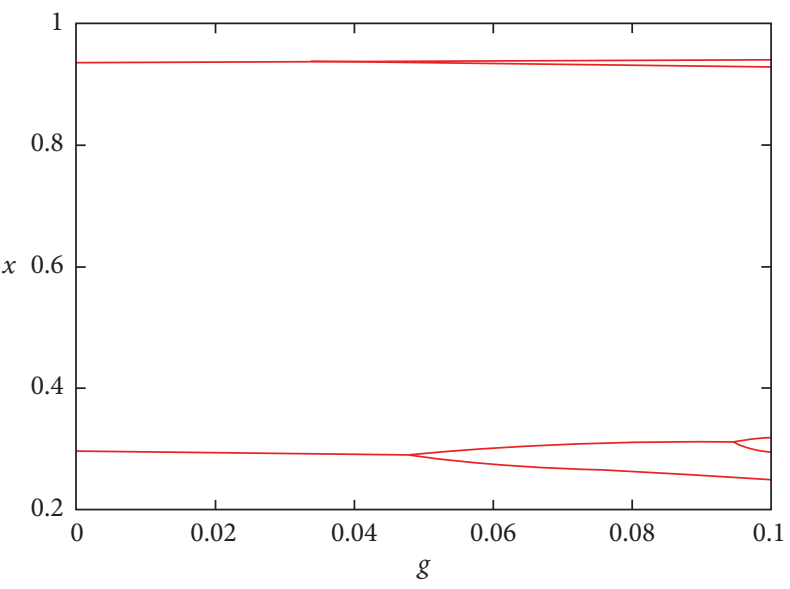

(b)

FiguRE 10: Bifurcation diagram and its corresponding Lyapunov exponent of nonlinear chemical reaction with $\omega=0.0481, \Omega=80 \omega$, $\alpha=0.0413, f=0.002$, and $\beta=0.0667$. Bifurcation diagrams and its corresponding Lyapunov exponent are obtained by scanning the parameter $g$ upwards (a) and downwards (b).

attractors. In addition, the simulations (the figures of which are not shown here) have shown that the parameters $\beta$ and $f$ reduce the two phenomena and also act on the chemical oscillations of the model studied. Figure 14 represents the Poincaré section of nonlinear chemical reaction and shows the $3 T$-periodic, $4 T$-periodic, and chaotic oscillations as also obtained in Figure 13. Finally, we look for the effect of the AM force when the frequencies of the amplitude modulation force are not resonant and such that their relationship to each other is irrational. 


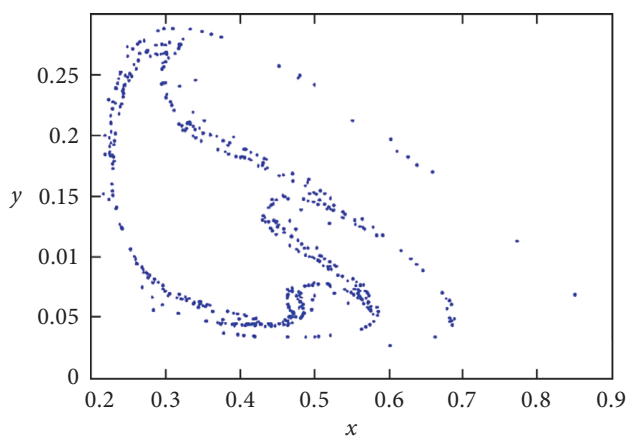

(a)

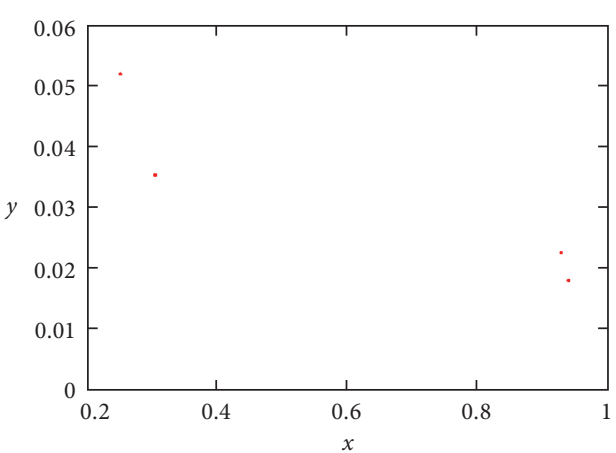

(b)

FIGURE 11: Poincaré section of nonlinear chemical reaction with $g=0.09$ and other parameters of Figure 10: (a) chaotic attractor for initial conditions $(0.1,0.1)$; (b) attractor of period $4 T$ for initial conditions $(0.5,0.5)$.

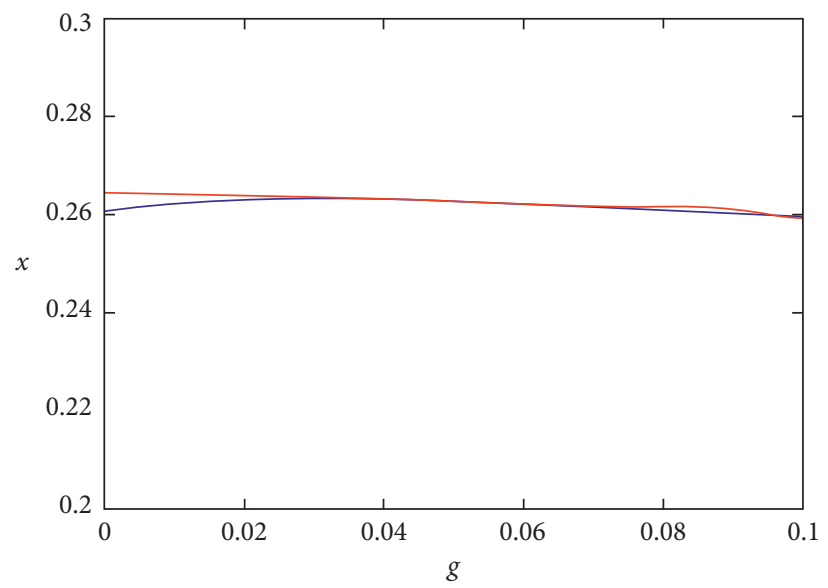

(a)

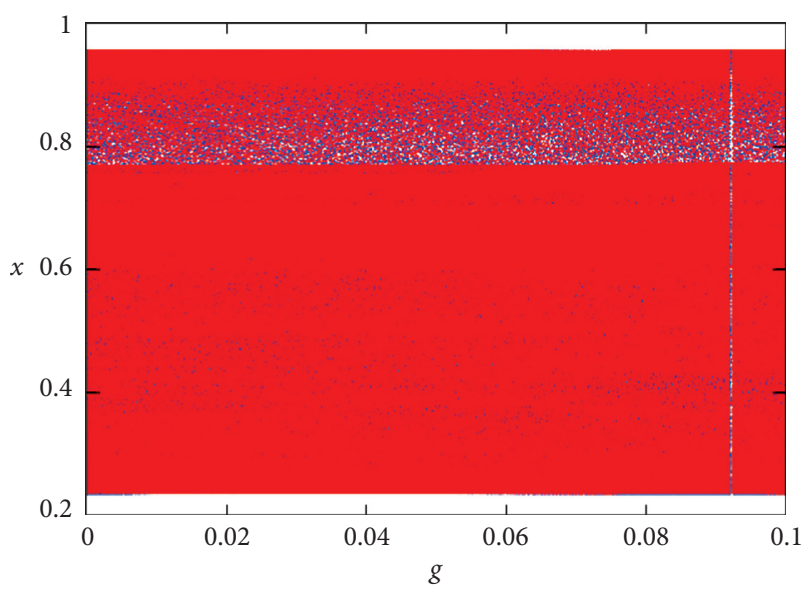

(b)

FIGURE 12: Effect of $\beta$ on bifurcation diagram and its corresponding Lyapunov exponent of nonlinear chemical reaction with $\omega=0.0481$, $\Omega=80 \omega, \alpha=0.0413$, and $f=0.002$ : (a) $\beta=0.04$; (b) $\beta=0.08$. Bifurcation diagrams and its corresponding Lyapunov exponent are obtained by scanning the parameter $g$ upwards (blue) and downwards (red).

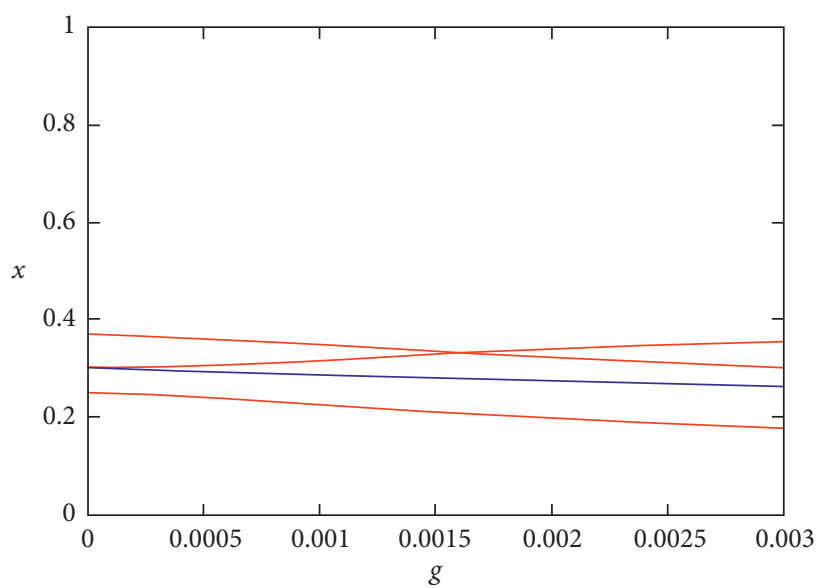

(a)

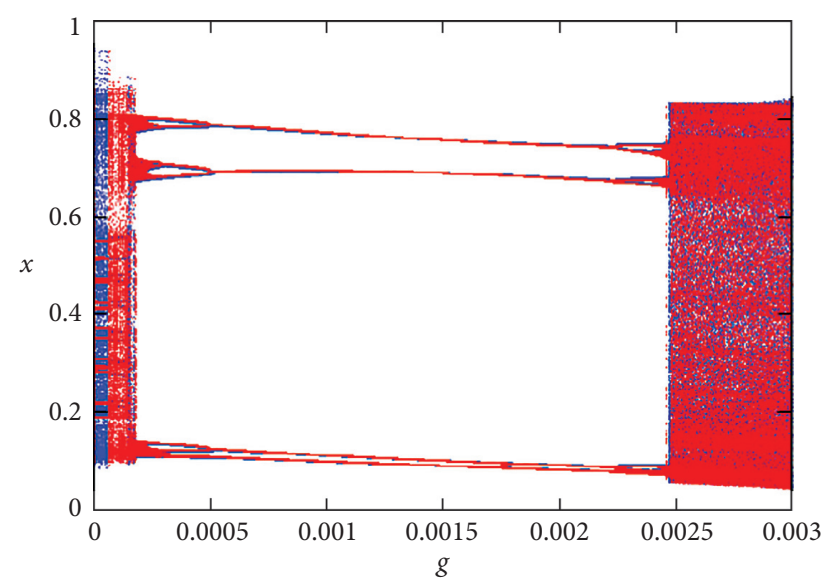

(b)

Figure 13: Continued. 


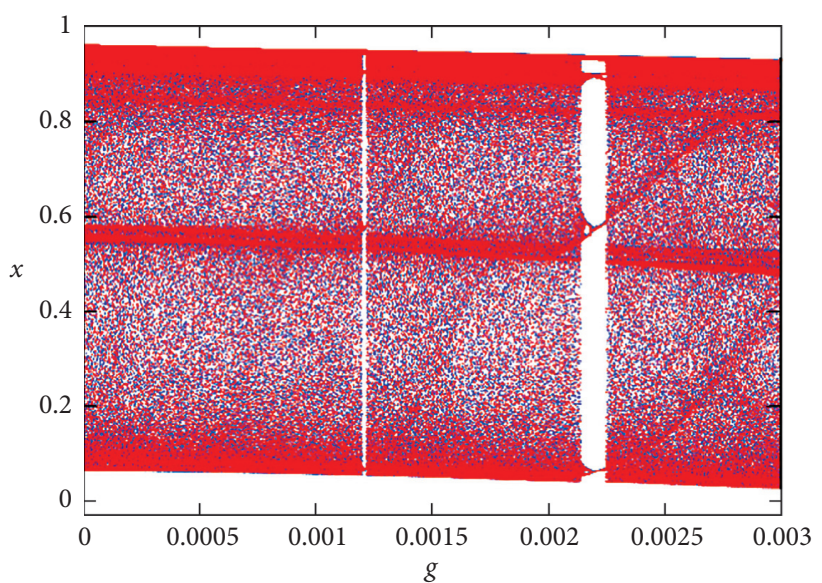

(c)

FIGURE 13: Effect of parameter $\alpha$ on bifurcation diagram of nonlinear chemical reaction with $\Omega=\omega=0.0481, f=0.002$, and $\beta=0.04$ : (a) $\alpha=0.03$; (b) $\alpha=0.028$; (c) $\alpha=0.02$. Bifurcation diagrams and its corresponding Lyapunov exponent are obtained by scanning the parameter $g$ upwards (blue) and downwards (red).

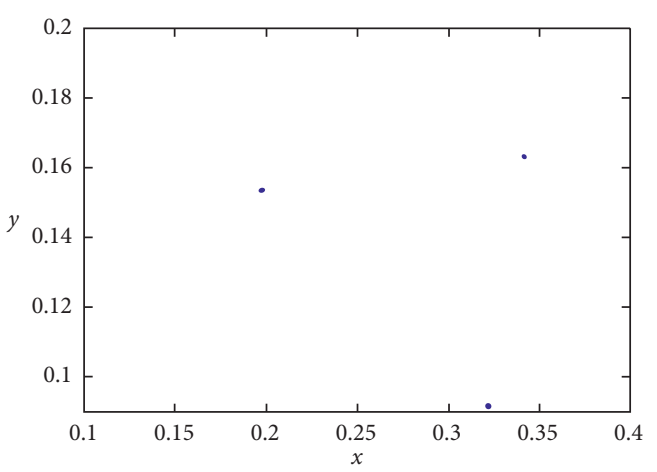

(a)

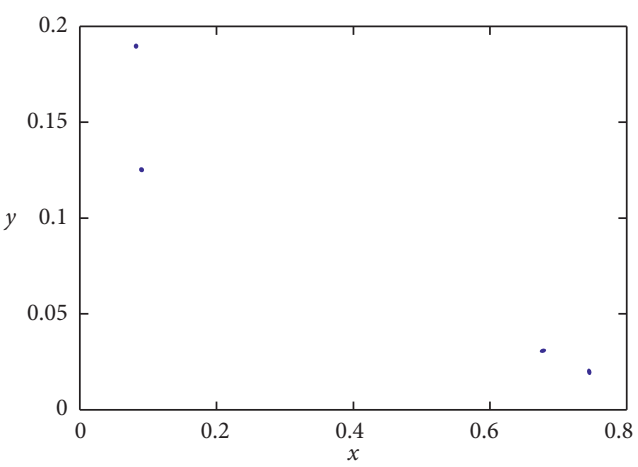

(b)

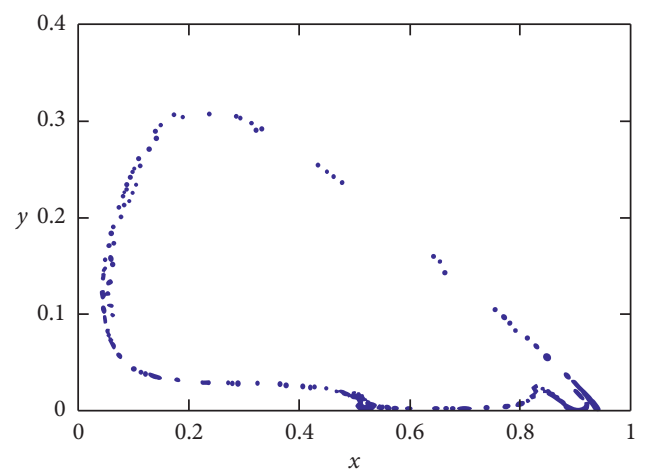

(c)

Figure 14: Effect of $\alpha$ on Poincaré section of nonlinear chemical reaction with $g=0.002$ and other parameters of Figure 13: (a) $\alpha=0.03$ : attractor of period 3T; (b) $\alpha=0.028$ : attractor of period $4 T$; (c) $\alpha=0.02$ : chaotic attractor.

For this, we take $\omega=0.0481$ and $\Omega=(\sqrt{5}-1) / 2$; we also seek the dynamics of the system by constructing the bifurcation diagram considering $g$ as control parameter. Figures 15 and 16, respectively, represent the effects of $\beta$ and $f$ on the bifurcation diagram. When we analyze these figures, we note the same remarks as the case where $\omega=\Omega$ except that here each of the parameters $\beta$ and $f$ can be used to reduce or even eliminate the chaotic chemical oscillations. 


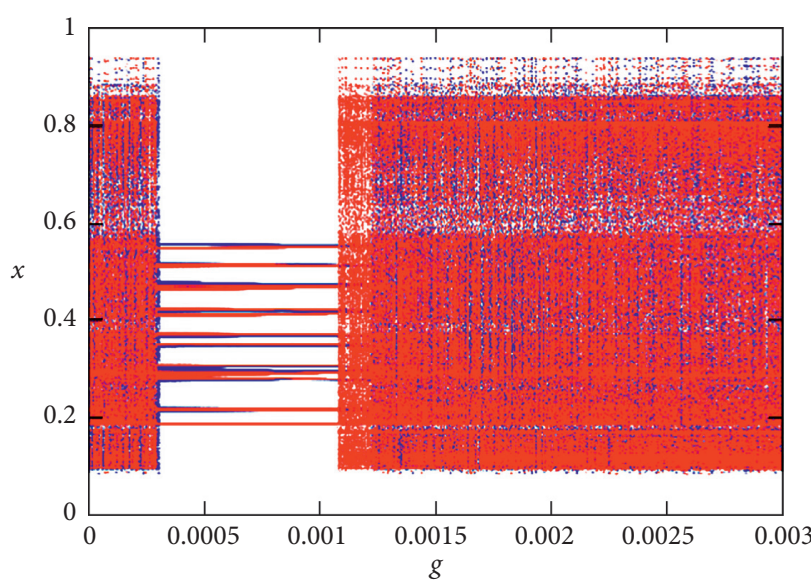

(a)

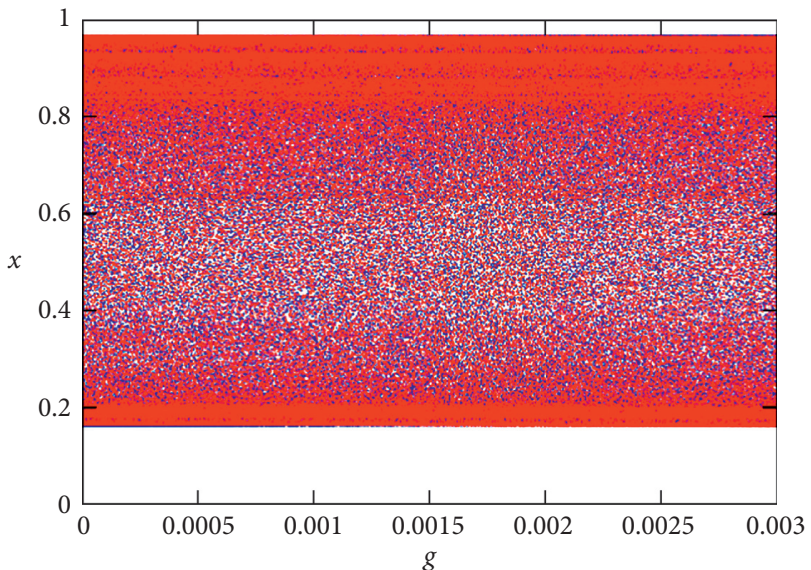

(b)

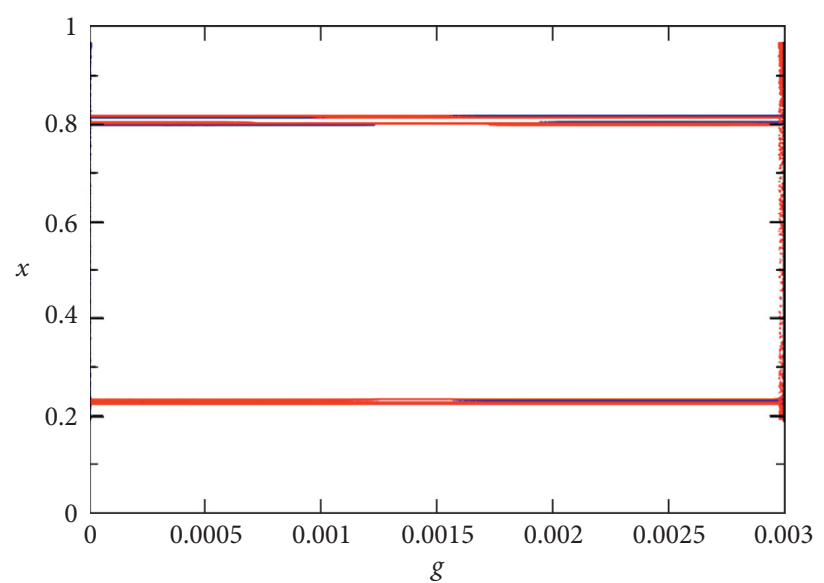

(c)

FIGURE 15: Effect of parameter $\beta$ on bifurcation diagram of nonlinear chemical reaction with $\omega=0.0481, \Omega=(\sqrt{5}-1) / 2, f=0.002$, and $\alpha=0.28$ : (a) $\beta=0.04$; (b) $\beta=0.06$; (c) $\beta=0.07$. Bifurcation diagrams and its corresponding Lyapunov exponent are obtained by scanning the parameter $g$ upwards (blue) and downwards (red).

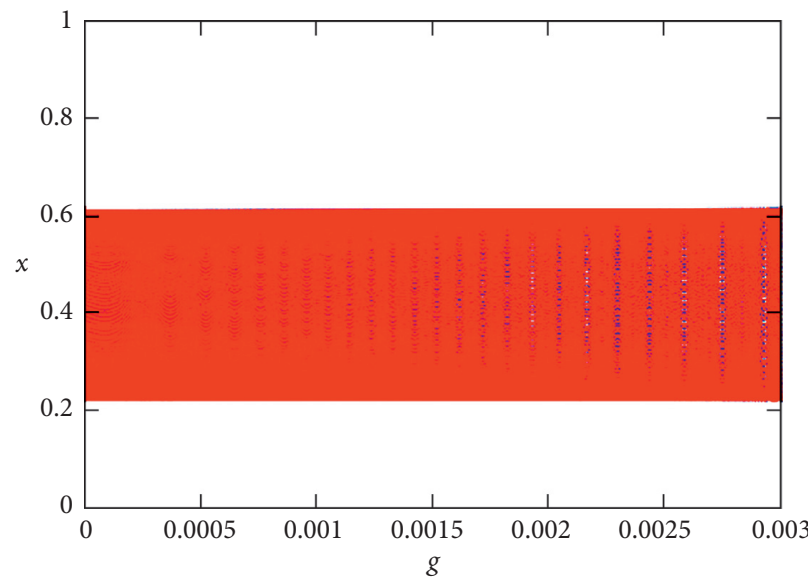

(a)

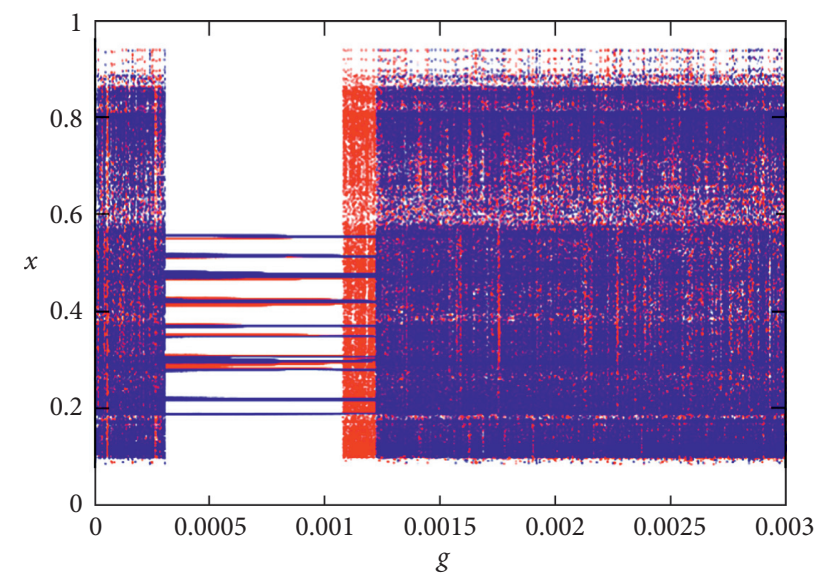

(b)

Figure 16: Continued. 


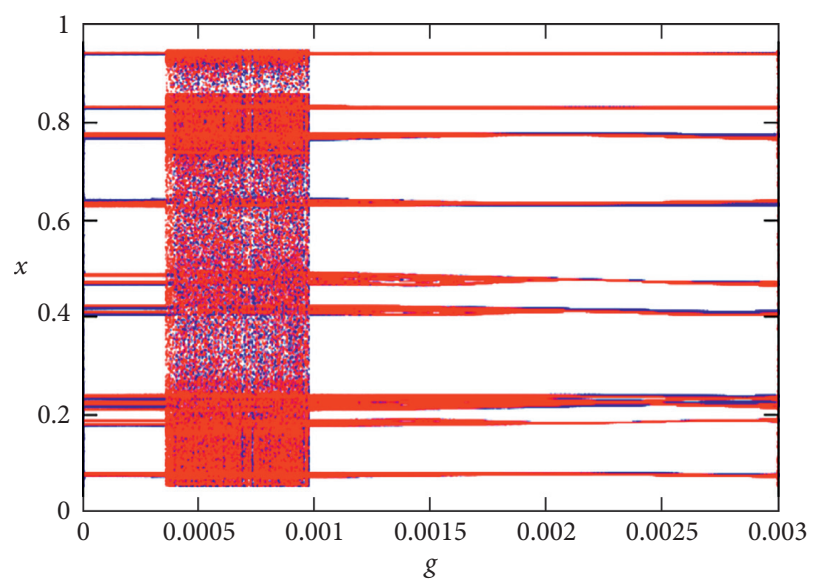

(c)

FigURE 16: Effect of parameter $f$ on bifurcation diagram of nonlinear chemical reaction with $\omega=0.0481, \Omega=(\sqrt{5}-1) / 2, \alpha=0.28$, and $\beta=0.04$ : (a) $f=0$; (b) $f=0.002$; (c) $f=0.0025$. Bifurcation diagrams and its corresponding Lyapunov exponent are obtained by scanning the parameter $g$ upwards (blue) and downwards (red).

\section{Conclusions}

In this work, we have studied the influence of an amplitudemodulated force on the chemical oscillations of reactions between four molecules. We have looked for fixed points and their natures when the system is autonomous. It appears that the autonomous system admits exactly four fixed points. The nature of the nontrivial fixed point depends of parameters $\alpha$ and $\beta$, and Hopf bifurcation is obtained for these two parameters for the nontrivial equilibrium point. The dynamics of the chemical system considered has been widely analyzed in the unmodulated case, and periodic, multiperiodic, quasiperiodic, and chaotic chemical oscillations have been obtained. Domains of existence of hysteresis, of coexistence of quasiperiodic attractors with attractors of period $5 T$ and attractors of period $6 T$, and of coexistence of multiperiodic attractors with chaotic attractors have been obtained. From the simulations made, it should be noted that, for certain values of the nonlinear parameters $\alpha$ and $\beta$, the complex dynamics can be controlled and even reduced to a periodic oscillation. For $g 6=0$ and for $\Omega \omega$, complex phenomena such as vibrational resonance (VR), hysteresis, and coexistence of attractors appear and are very remarkable and can be well controlled either by the AM force or by the parameters $\alpha$ and $\beta$. The domains of existence of these different phenomena are less important when $\Omega=\omega$. When the frequencies of the amplitude modulation force are not resonant and are such that their relationship to each other is irrational, the multiperiodic attractors when they exist are of period $\geq 4 T$ and their domains of existence are more important. Finally, our work confirmed the chaotic oscillation and the Hopf bifurcation obtained in [5] and proved that the appearance of VR, hysteresis, multistability, and coexistence of various attractors in the chemical reaction is considered. The presence of VR shows that the amplitude of oscillations of concentrations can be explored, while the hysteresis phenomenon indicates that the amplitude of oscillations of concentrations will not be able to follow the same path of evolution when the control parameter increases or decreases following the same path. The multistability and the coexistence of attractors obtained reveal that the stable or unstable nature of the chemical oscillations and of the chemical attractors in the same domain of the parameters is considered. The use of the amplitude modulated force has shown that the various complex phenomena obtained can be controlled.

\section{Data Availability}

No data were used in this study.

\section{Conflicts of Interest}

The authors declare that there are no conflicts of interest regarding the publication of this paper.

\section{References}

[1] S. Strogatz, Nonlinear Dynamics and Chaos: With Applications to Physics, Biology, Chemistry, and Engineering, Ingram Publisher Services US, New York, NY, USA, 2nd edition, 2014.

[2] H. Binous and A. Bellagi, "Introducing nonlinear dynamics to chemical and biochemical engineering graduate students using Mathematica," Computer Applications in Engineering Education, vol. 27, no. 1, pp. 1-19, 2018.

[3] S. Guruparan, B. Ravindran Durai Nayagam, V. Ravichandran, V. Chinnathambi, and S. Rajasekar, "Hysteresis, vibrational resonance and chaos in brusselator chemical system under the excitation of amplitude modulated force," Chemical Science Review and Letters, vol. 4, no. 15, pp. 870-879, 2015.

[4] M. Gruebelle and P. G. Wolynes, "Vibrational energy flow and chemical reactions," Accounts of Chemical Research, vol. 37, pp. 261-267, 2004.

[5] A. Shabunin, V. Astakhov, V. Demidov et al., "Modeling chemical reactions by forced limit-cycle oscillator: 
synchronization phenomena and transition to chaos," Chaos, Solitons \& Fractals, vol. 15, no. 2, pp. 395-405, 2003.

[6] I. I. Blekhman and P. S. Landa, "Conjugate resonances and bifurcations in nonlinear systems under biharmonical excitation," International Journal of Non-linear Mechanics, vol. 39, no. 3, pp. 421-426, 2004.

[7] T. O. Roy-Layinde, J. A. Laoye, O. O. Popoola, and U. E. Vincent, "Analysis of vibrational resonance in biharmonically driven plasma," Chaos, vol. 26, pp. 1-9, 2016.

[8] P. S. Landa and P. V. E. McClintock, "Vibrational resonance," Journal of Physics A: Mathematical General, vol. 33, no. 45, pp. 433-438, 2000.

[9] C. Jeevarathinam, S. Rajasekar, and M. A. F. Sanju'an, "Vibrational resonance in the Duffing oscillator with distributed time-delayed feedback," Journal of Applied Nonlinear Dynamics, vol. 4, pp. 1-15, 2015.

[10] P. Sarkar and D. S. Ray, "Vibrational antiresonance in nonlinear coupled systems," Physical Review E, vol. 99, no. 52221, pp. 1-7, 2019.

[11] D. L. Olabodé, C. H. Miwadinou, V. A. Monwanou, and J. B. Chabi Orou, "Effects of passive hydrodynamics force on harmonic and chaotic oscillations in nonlinear chemical dynamics," Physica D, vol. 386387, pp. 49-59, 2019.

[12] C. H. Miwadinou, A. V. Monwanou, J. Yovogan, L. A. Hinvi, P. R. Nwagoum Tuwa, and J. B. Chabi Orou, "Modeling nonlinear dissipative chemical dynamics by a forced modified Van der Pol-Duffing oscillator with asymmetric potential: chaotic behaviors predictions," Chinese Journal of Physics, vol. 56, no. 3, pp. 1089-1104, 2018.

[13] H. G. Enjieu Kadji and B. R. Nana Nbendjo, "Passive aerodynamics control of plasma instabilities," Communications in Nonlinear Science and Numerical Simulation, vol. 17, no. 4, pp. 1779-1794, 2012.

[14] C. H. Miwadinou, A. V. Monwanou, L. A. Hinvi, and J. B. Chabi Orou, "Effect of amplitude modulated signal on chaotic motions in a mixed Rayleigh-Liénard oscillator," Chaos, Solitons \& Fractals, vol. 113, pp. 89-101, 2018.

[15] C. H. Miwadinou, A. V. Monwanou, A. A. Koukpemedji, Y. J. F. Kpomahou, and J. B. Chabi Orou, "Chaotic motions in forced mixed Rayleigh-Li' enard oscillator with external and parametric periodic excitations," International Journal of Bifurcation and Chaos, vol. 28, no. 3, pp. 1-16, 2018.

[16] A. Shabunin, F. Baras, and A. Provata, "Oscillatory reactive dynamics on surfaces: a lattice limit cycle model," Physical Review E, vol. 66, pp. 1-11, 2002.

[17] G. Nicolis and I. Prigogine, Self-organization in Nonequilibrium Systems, Wiley, New York, NY, USA, 1977.

[18] R. Imbihl and G. Ertl, "Oscillatory kinetics in heterogeneous catalysis," Chemical Reviews, vol. 95, no. 3, pp. 697-733, 1995.

[19] C. G. Takoudis, L. D. Schmidt, and R. Aris, "Isothermal sustained oscillations in a very simple surface reaction," Surface Science, vol. 105, no. 1, pp. 325-333, 1981.

[20] I. G. Kevrekidis, L. D. Schmidt, and R. Aris, "Some common features of periodically forced reacting systems," Chemical Engineering Science, vol. 41, no. 5, pp. 1263-1276, 1986.

[21] M. A. Taylor and I. G. Kevrekidis, "Some common dynamic features of coupled reacting systems," Physica D: Nonlinear Phenomena, vol. 51, no. 1-3, pp. 274-292, 1991.

[22] C. Hayashi, Nonlinear Oscillations in Physical Systems, McGraw-Hill, New York, NY, USA, 1964.

[23] A. H. Nayfeh, Introduction to Perturbation Techniques, John Wiley \& Sons, New York, NY, USA, 1981. 